\title{
Ka and Q band Propagation Experiments in Toulouse using ASTRA 3B and ALPHASAT Satellites
}

\author{
Xavier Boulanger ${ }^{1}$ and Laurent Castanet $^{1}$ \\ ${ }^{1}$ ONERA - Université Fédérale de Toulouse (UFTMiP) - ElectroMagnetics \& Radar Department, \\ Propagation, Environment and Radio-communications research unit - BP 74025, 2 Avenue Edouard Belin, \\ 31055 Toulouse Cedex 4, France
}

\begin{abstract}
SUMMARY
From January 2008 to March 2011, ONERA operated in Toulouse, France, a beacon receiver able to collect the $19.7 \mathrm{GHz}$ beacon signal of the HotBird 6 satellite. In March 2011, the RF chain was modified to be able to receive the $20.2 \mathrm{GHz}$ ASTRA 3B beacon to benefit from a higher EIRP of the satellite. Since June 2015, a second beacon receiver has been installed and is able to record the $39.4 \mathrm{GHz}$ beacon signal of the Alphasat satellite.

The objective of this paper is to present the results of the first two years of measurements (June 2015-May 2017) of the Q-band Alphasat propagation experiment in Toulouse, as well as concurrent measurements collected at Ka-band with ASTRA 3B. First of all, the propagation experiments are briefly described. Secondly, the results of the statistical analysis are highlighted. Finally, a deep analysis of the performances of the two frequency scaling prediction methods recommended in ITU-R P.618-12 is performed.
\end{abstract}

KEY WORDS: Alphasat, ASTRA 3B, Attenuation, Frequency Scaling, Propagation, Satellite Communications.

\section{INTRODUCTION}

The Alphasat satellite was launched in July 2013. Two beacons, one at Ka-band and one at Q-band, constitute the Alphasat Aldo Paraboni scientific payload [1], [2]. Both signals are used by the propagation community around Europe for collecting propagation statistics [3]. The collaborative Group of the AlphaSat Aldo Paraboni propagation Experimenters (ASAPE) ensures the collaboration between all research organizations for the execution of the campaigns, data processing and analysis to test propagation models.

Over the past decade, ONERA has been involved into several Earth-space propagation experiments. From January 2008 to March 2011, ONERA operated in Toulouse, France, a beacon receiver able to collect the $19.7 \mathrm{GHz}$ beacon signal of the HotBird 6 satellite [4]. In March 2011, the RF chain was modified to be able to receive the 20.2 GHz ASTRA 3B beacon [4], [5]. From mid-2013 to end of 2014, ONERA and CNES deployed four other Ka-band beacon receivers in the South of France in order to assess the performances of Site Diversity techniques [5]. Since June 2015, a Q-band beacon receiver has been installed in Toulouse (co-located to the first ASTRA 3B beacon receiver) and is able to record the $39.4 \mathrm{GHz}$ beacon signal of the Alphasat satellite [6]. 
Section 2 of this paper is dedicated to the description of the $\mathrm{Ka}$ and $\mathrm{Q}$ band propagation experiments. The link parameters as well as the experimental set-up are introduced. The results of the statistical analysis, in particular the monthly and annual fluctuations at Q-band, are highlighted in Section 3. Finally, as concurrent measurements at Ka-band are available (using the co-located ASTRA 3B beacon receiver), a deep analysis of the performances of the two frequency scaling prediction methods recommended in ITUR P.618-12 [7] is performed. The results are shown in Section 4.

\section{DESCRIPTION OF THE KA AND Q BAND PROPAGATION EXPERIMENTS}

\subsection{Main characteristics}

The $\mathrm{Ka}$ and $\mathrm{Q}$ band propagation experiments are performed on the rooftop of the Electromagnetism and Radar Department building of the ONERA Toulouse Centre, France, located at $43.5710^{\circ} \mathrm{N}$ of latitude and $1.4716^{\circ} \mathrm{E}$ of longitude (Figure 1). Situated in the South-West of France, Toulouse is subject to both Atlantic Ocean and Mediterranean Sea influences. The climate normals, computed over the period 1981-2010, are the following [8]: the mean annual temperature is $13.8^{\circ} \mathrm{C}$, the mean annual minimum temperature is $9.1^{\circ} \mathrm{C}$ and the mean annual maximum temperature is $18.5^{\circ} \mathrm{C}$. The mean number of rainy days is 95.7 days/year and the mean rainfall amount is $638.3 \mathrm{~mm} /$ year. The precipitations are not homogeneously distributed all over the months and remain between $37.7 \mathrm{~mm}$ in July (the driest month) and $74 \mathrm{~mm}$ in May (the wettest month).

At the end of March 2011, the Ka band beacon receiver previously used for the HotBird 6 experiment was upgraded to benefit from the better EIRP of the SES ASTRA 3B $\mathrm{Ka}$ band beacon [4], [5]. ASTRA 3B is located on a geostationary orbit at $23.5^{\circ} \mathrm{E}$ of longitude. The signal source is the $20.199 \mathrm{GHz}$ beacon (vertical polarization) with a beacon EIRP of $28.8 \mathrm{dBW}$ over Toulouse. The azimuth and elevation angles from Toulouse are respectively $149.57^{\circ}$ and $35.11^{\circ}$. The signal level is recorded at a $10 \mathrm{~Hz}$ sampling rate and the dynamic range of the beacon receiver is around $37 \mathrm{~dB}$ for a $50 \mathrm{~Hz}$ receiver bandwidth.

Since June 2015, a Q-band beacon receiver has been installed in Toulouse (colocated to the first ASTRA 3B beacon receiver) and is able to record the $39.402 \mathrm{GHz}$ (linear tilted $45^{\circ}$ polarization) beacon signal of the Alphasat satellite [6]. Alphasat is located on an inclined geostationary orbit (maximum $\pm 3^{\circ}$ ) at $25^{\circ} \mathrm{E}$ of longitude. The Alphasat $\mathrm{Q}$ band beacon EIRP is about $26 \mathrm{dBW}$ over Toulouse. The mean azimuth and elevation angles from Toulouse are respectively $147.70^{\circ}$ and $34.49^{\circ}$. The signal level is also recorded at a $10 \mathrm{~Hz}$ sampling rate and the dynamic range of the beacon receiver is around $30 \mathrm{~dB}$ for a $50 \mathrm{~Hz}$ receiver bandwidth. 


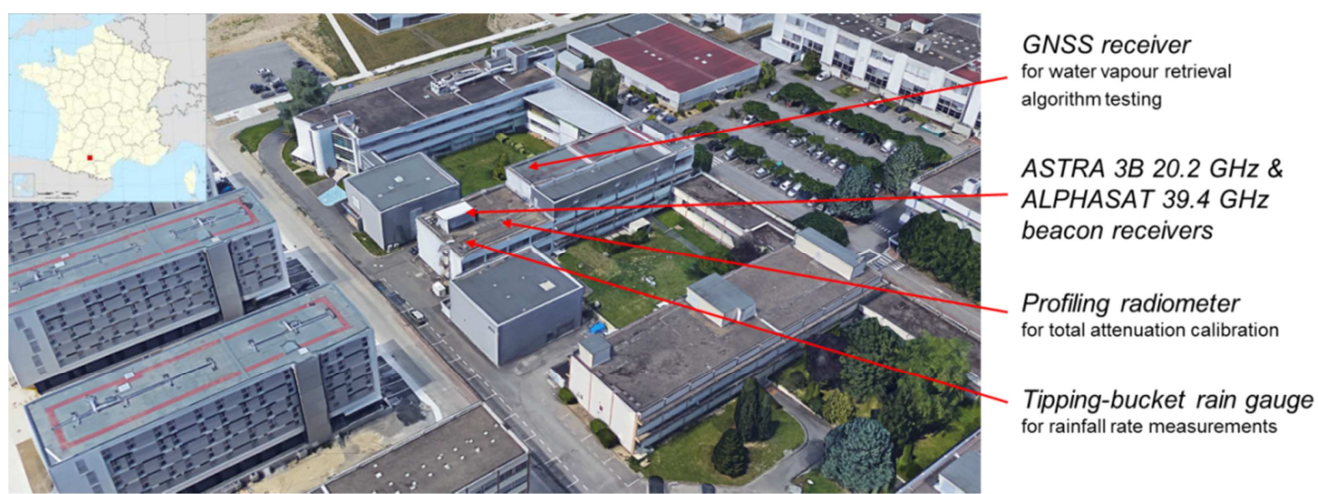

Figure 1. Overview of the Ka and Q band propagation experiments in Toulouse, France

\subsection{Experimental set-up}

The experimental set-up of the Ka and Q band propagation experiments in Toulouse is the following:

- An indoor Ka-band beacon receiver,

- An indoor Q-band beacon receiver,

- A profiling radiometer for total attenuation calibration,

- A tipping-bucket rain gauge for rainfall rate measurements,

- A GNSS receiver for water vapour retrieval algorithm testing (not described in this paper as out of the scope).

They are briefly described in the following sections.

\subsubsection{Ka and $Q$ band beacon receivers}

At the end of the 90's, ONERA designed and built two indoor beacon receivers (20.7 and $41.4 \mathrm{GHz}$ ) for the Stentor propagation experiment [9]. After the launch failure and the loss of the satellite, the $20.7 \mathrm{GHz}$ beacon receiver was modified to receive firstly the $19.7 \mathrm{GHz}$ Hotbird 6 beacon [4] and secondly the 20.2 GHz ASTRA 3B beacon [4], [5]. The Alphasat mission has given the opportunity to work at higher frequency with the Qband beacon of the Alphasat Aldo Paraboni Scientific payload. Consequently, the second beacon receiver has been slightly upgraded to receive the $39.4 \mathrm{GHz}$ Alphasat beacon signal [6].

The main characteristics of the two indoor beacon receivers are very similar. Basically, they consist of a Cassegrain parabolic antenna of $1.20 \mathrm{~m}$ diameter with the RF chain immediately placed at the output of the antenna to avoid feeder loss. Three successive frequency down-conversions are performed:

- a first one from $20.2 \mathrm{GHz}$ to $110 \mathrm{MHz}$ or from $39.4 \mathrm{GHz}$ to $220 \mathrm{MHz}$ for the $\mathrm{Ka}$ and $\mathrm{Q}$ band beacon receivers respectively; 
- a second one from 110 to $10 \mathrm{MHz}$ or from 220 to $20 \mathrm{MHz}$ for the $\mathrm{Ka}$ and Q band beacon receivers respectively,

- a third one from $10 \mathrm{MHz}$ to $2 \mathrm{kHz}$ or from $20 \mathrm{MHz}$ to $2.5 \mathrm{kHz}$ for the Ka and Q band beacon receivers respectively.

The Alphasat beacon receiver is equipped with a dynamic tracking system to counteract orbit inclination. It is based on the Orbit Ephemeris Messages files distributed by the ASAPE Group. The resolutions of the azimuth and elevation actuators are respectively $0.003^{\circ}$ and $0.002^{\circ}$. The reachable ranges are $11^{\circ}$ for the azimuth actuator and $6.2^{\circ}$ for the elevation actuator. The positions of both actuators are updated every minute. The ASTRA 3B beacon receiver has no dynamic tracking system (the antenna is only mechanically pointed once) as the satellite is relatively stable on its orbit. Figure 2 shows a picture of the co-located $\mathrm{Ka}$ and $\mathrm{Q}$ band beacon receivers deployed at Toulouse ONERA Centre premises.

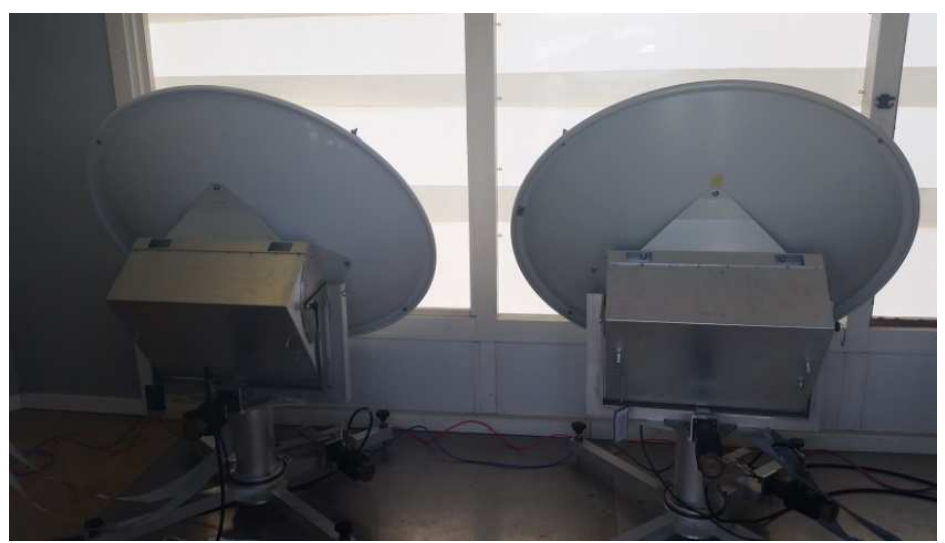

Figure 2. Co-located ASTRA 3B (right) and Alphasat (left) beacon receivers deployed at Toulouse ONERA Centre premises.

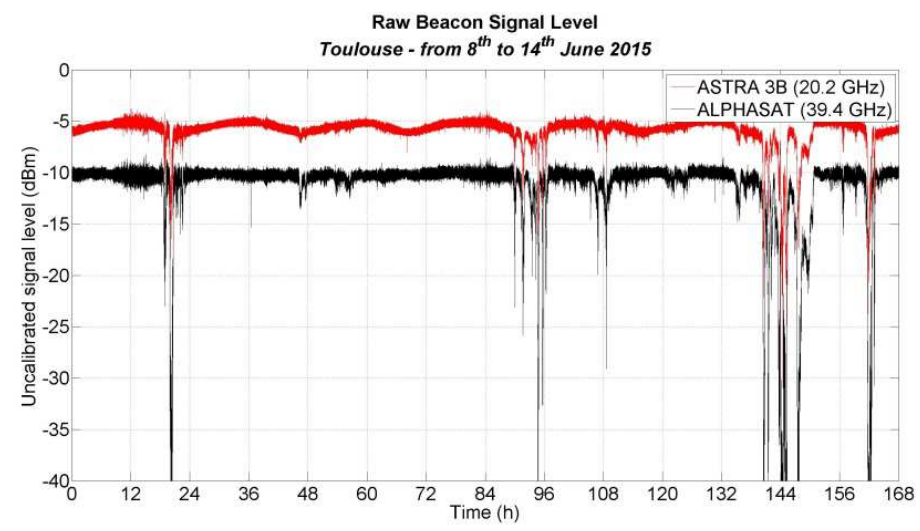

Figure 3. Raw beacon signal level recorded by the ASTRA 3B and Alphasat beacon receivers from $8^{\text {th }}$ to $14^{\text {th }}$ June 2015 . 
An example of raw (and uncalibrated) signal level recorded by both beacon receivers over one week (from $8^{\text {th }}$ to $14^{\text {th }}$ June 2015) is shown in Figure 3. A daily oscillation, mainly due to the slight satellite motion, can be observed on the ASTRA 3B beacon signal level. The peak-to-peak variation depends on the time of the initial pointing manoeuvre. The clear stability of the Alphasat beacon signal level proves that the tracking system works properly.

\subsubsection{Profiling microwave radiometer}

A microwave radiometer is often used in propagation experiments to calibrate the reference level of total attenuation in absorbing conditions [10], [11]. The one deployed at Toulouse ONERA Centre premises is a TP/WVP-3000 multi-frequency profiling radiometer built by Radiometrics (USA) [12], [13] which measures sky brightness temperatures at 12 frequency channels [4]:

- 5 in Ka-band between 22 and $30 \mathrm{GHz}$ around the water vapour absorption lines.

- 7 in V-band between 52 and $59 \mathrm{GHz}$ corresponding to the oxygen absorption lines.

In the $\mathrm{Ka}$ and $\mathrm{Q}$ bands experiments, the radiometer is mainly used to infer total attenuation, Integrated Water Vapour Content (IWVC), and Integrated Liquid Water Content (ILWC) in absorbing conditions on the slant path. The computation of the reference level of total attenuation in absorbing conditions relies on the Radiative Transfer Equation and on the estimation of the mean radiating (or effective) temperature $T_{m r}$ [14]. For each radiometer frequency $f_{i}^{\text {rad }}$ and a given elevation angle $\theta$, the radiometric attenuation $A_{\text {rad }}\left(f_{i}^{\text {rad }}, \theta\right)$ can be retrieved from [14]:

$$
A_{\text {rad }}\left(f_{i}^{\text {rad }}, \theta\right)=10 \cdot \log _{10}\left[\frac{T_{m r}\left(f_{i}^{r a d}, \theta\right)-T_{c}}{T_{m r}\left(f_{i}^{r a d}, \theta\right)-T_{b}\left(f_{i}^{r a d}, \theta\right)}\right]
$$

where $T_{c}$ is the temperature of the cosmic microwave background equal to $2.73 \mathrm{~K}$ [15] and $T_{b}\left(f_{i}^{r a d}, \theta\right)$ is the brightness temperature.

Nevertheless, the beacon frequency $f_{b}$ generally does not match any of the radiometer frequencies. So, the reference level of total attenuation in absorbing conditions can be approximated from the following linear regression [10], [11]:

$$
A_{\text {rad }}\left(f_{b}, \theta\right)=a_{0}+\sum_{f_{i}^{\text {rad }}} a_{i} \cdot A_{\text {rad }}\left(f_{i}^{\text {rad }}, \theta\right)
$$

where the coefficients $a_{i}$ depend on the elevation, the location and the number of frequencies taken into account. Radiosounding (RAOBS) profiles representative of the sites where the beacon measurements are carried out are often used as input of a Radiative Transfer Simulator to retrieve those coefficients. In a similar way, IWVC and ILWC can be inferred from: 


$$
\begin{aligned}
& \operatorname{IWVC}(\theta)=b_{0}+\sum_{f_{i}^{\text {rad }}} b_{i} \cdot A_{\text {rad }}\left(f_{i}^{\text {rad }}, \theta\right) \\
& \operatorname{ILWC}(\theta)=c_{0}+\sum_{f_{i}^{\text {rad }}} c_{i} \cdot A_{\text {rad }}\left(f_{i}^{\text {rad }}, \theta\right)
\end{aligned}
$$

For both ASTRA 3B Ka-band and Alphasat Q-band propagation experiments, monthly slant path ( $35^{\circ}$ of elevation) coefficients $a_{i}$ (for ASTRA 3B) and $a^{\prime}{ }_{i}$ (for Alphasat) have been derived using radiosounding profiles collected in Bordeaux $\left(44.84^{\circ} \mathrm{E},-0.58^{\circ} \mathrm{E}, 200 \mathrm{~km}\right.$ away from Toulouse) and four radiometric channels $(23.84,30,51.25$, and $52.28 \mathrm{GHz})$ leading to :

$$
\begin{aligned}
& A_{20.2}=a_{0}+a_{1} A_{23.84}+a_{2} A_{30}+a_{3} A_{51.25}+a_{4} A_{52.28} \\
& A_{39.4}=a_{0}^{\prime}+a_{1}^{\prime} A_{23.84}+a_{2}^{\prime} A_{30}+a^{\prime}{ }_{3} A_{51.25}+a_{4}^{\prime} A_{52.28}
\end{aligned}
$$

Monthly slant path ( $35^{\circ}$ of elevation) coefficients $b_{i}$, and $c_{i}$ have also been derived using the same radiosounding database and three radiometric channels $(23.04,23.84$, and $30 \mathrm{GHz})$ leading to:

$$
\begin{aligned}
& I W V C=b_{0}+b_{1} A_{23.04}+b_{2} A_{23.84}+b_{3} A_{30} \\
& I L W C=c_{0}+c_{1} A_{23.04}+c_{2} A_{23.84}+c_{3} A_{30}
\end{aligned}
$$

For the sake of conciseness, tables of coefficients will be not presented in this paper. Finally, the full methodology described in [4] is then used to combine radiometric and beacon measurements to retrieve calibrated time series of rain and total attenuation. An example of calibrated time series of total attenuation is shown in Figure 4.

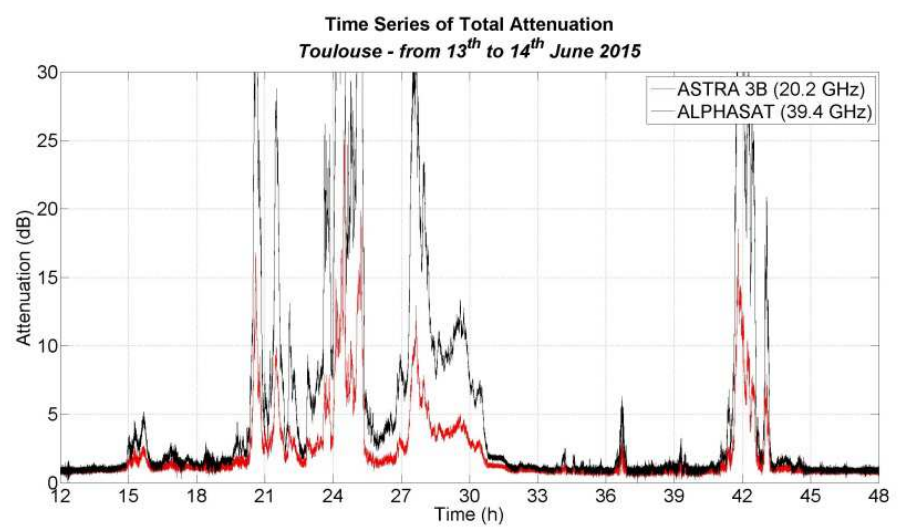

Figure 4. Calibrated time series of total attenuation from $13^{\text {th }}$ to $14^{\text {th }}$ June 2015

\subsubsection{Tipping-bucket rain gauge}

A corrosion resistant dual-chamber tipping bucket rain gauge able to record the exact instant of tips ( 1 second resolution) is also available. It was manufactured by FIEDLER, $\mathrm{CZ}$ and the main characteristics are: 
- collecting area of $500 \mathrm{~cm}^{2}$

- $0.1 \mathrm{~mm}$ per pulse resolution

- $0.015 \mathrm{~mm}$ accuracy

The rain gauge data are converted into rain rate measurements (in $\mathrm{mm} / \mathrm{h}$ ) according to the methodology described in [16]10.

\section{STATISTICAL ANALYSIS}

Two full years (June 2015 - May 2017) of data have been processed. Daily rain and total attenuation validated time series have been produced and statistical analyses have been performed. The availabilities of valid data used for the different statistical analyses are reported in Table 1.

Figure 5 shows the Complementary Cumulative Distribution Functions (CCDF) of concurrent rain and total attenuation at $20.2 \mathrm{GHz}$ and $39.4 \mathrm{GHz}$. A small year-to-year variability can be noticed. As an example, the total attenuation values at $20.2 \mathrm{GHz}$ exceeded for $1 \%$ of the time are $1.9 \mathrm{~dB}$ and $1.6 \mathrm{~dB}$ for the first and second year of measurements, respectively. At $39.4 \mathrm{GHz}$, the total attenuation values exceeded for $1 \%$ of the year are $4.3 \mathrm{~dB}$ and $3.3 \mathrm{~dB}$. For $0.1 \%$ of the time, the total attenuation values at $20.2 \mathrm{GHz}$ are $5.8 \mathrm{~dB}$ and $4.1 \mathrm{~dB}$ for the first and second year of measurements, respectively. At $39.4 \mathrm{GHz}$, the total attenuation values exceeded for $0.1 \%$ of the year are $17.6 \mathrm{~dB}$ and $11.4 \mathrm{~dB}$.

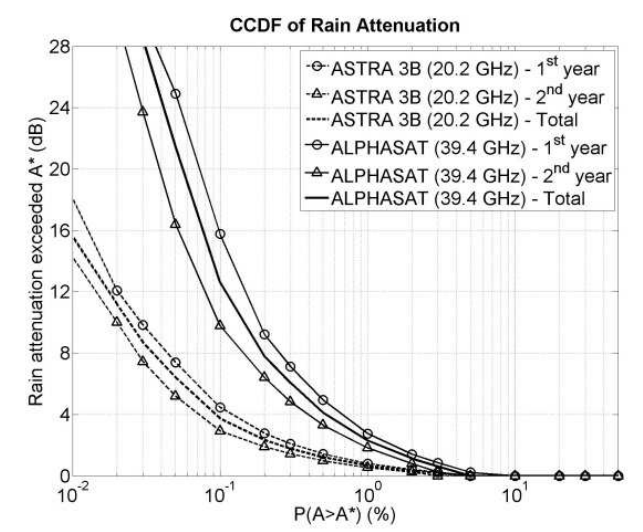

(a)

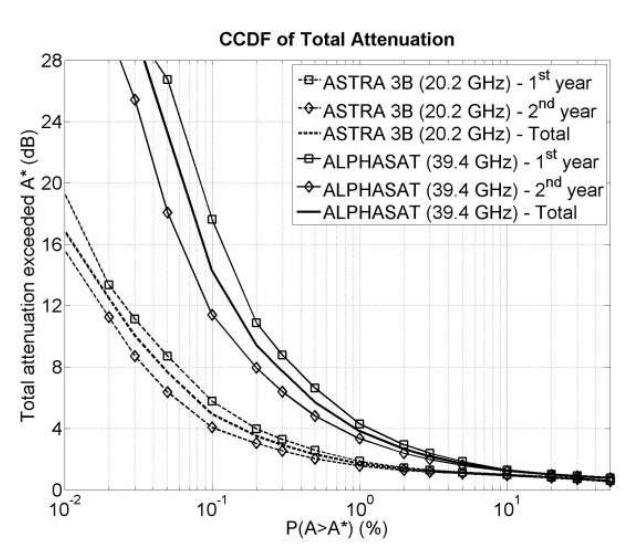

(b)

Figure 5. CCDF of concurrent attenuation at 20.2 and $39.4 \mathrm{GHz}$. (a) Rain attenuation. (b)

Total attenuation

Figure 6 shows CCDF of concurrent rainfall rate and rain attenuation at $39.4 \mathrm{GHz}$ for the full two-year period. It is worth noting that the dynamic range of the beacon receiver is reached for $0.03 \%$. The rainfall rate value exceeded for $0.01 \%$ of the average year is around $35 \mathrm{~mm} / \mathrm{h}$. 
Figure 7 shows the monthly CCDF of rain and total attenuation at $39.4 \mathrm{GHz}$. A high month-to-month variability can be noticed with the strongest rain attenuation (and so the less favourable propagation conditions) from May to August and the weakest rain attenuation in December and January.

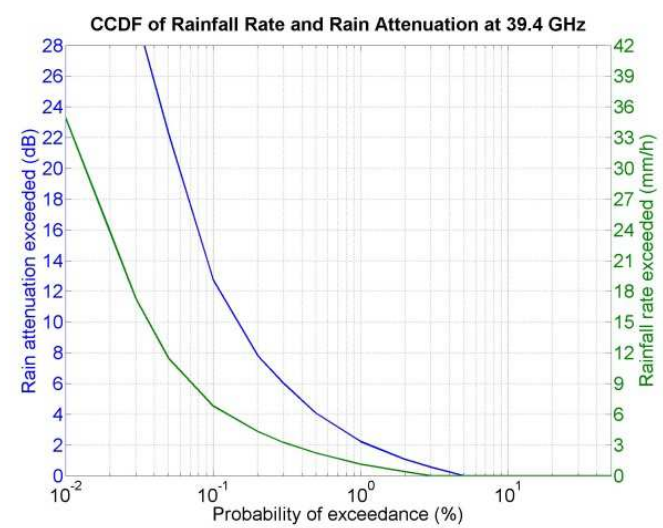

Figure 6. CCDF of concurrent rainfall rate and rain attenuation at $39.4 \mathrm{GHz}$ for the full two-year period

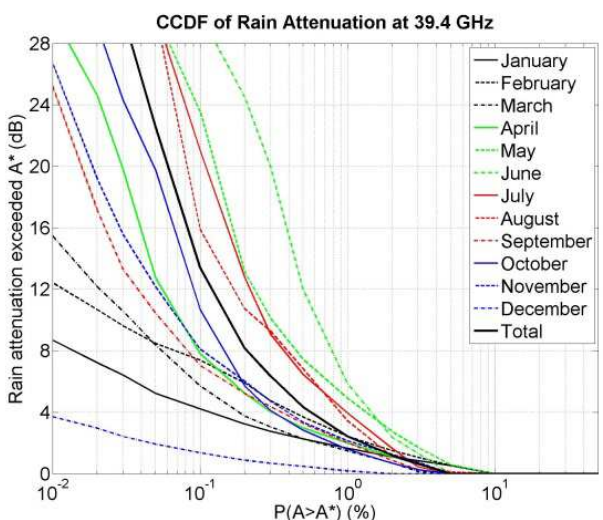

(a)

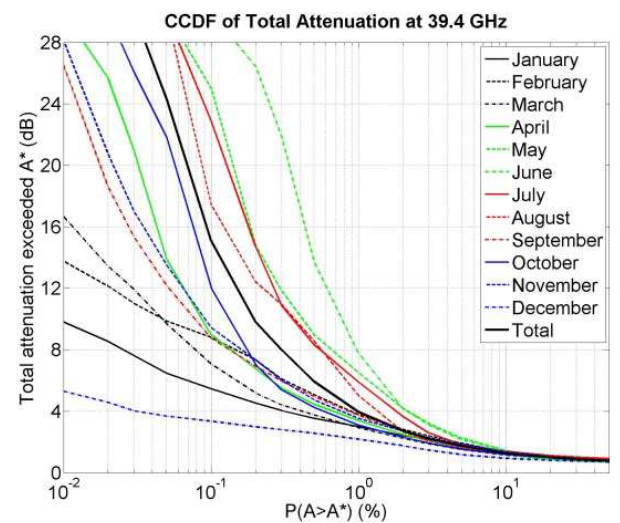

(b)

Figure 7. Monthly CCDF of attenuation at $39.4 \mathrm{GHz}$. (a) Rain attenuation. (b) Total attenuation

\section{FREQUENCY SCALING ANALYSIS}

Up-link power control (ULPC) is one of the most effective fade mitigation techniques (FMT) to increase link availability and service quality of satellite communication systems operating at very high frequencies (Ka-band and above) [17], [18], and [19]. It allows the Earth station transmit power to be adjusted with respect to the propagation conditions and so severe attenuation (mainly caused by rain in these frequency bands) to be counteracted. The estimation of the attenuation on the up-link can be 
performed using opportunistic signals (down-link, beacons ...) which often operate at lower frequencies than the up-link. The scaling from attenuation $A_{\text {down }}$ at frequency $f_{\text {down }}$ to attenuation $A_{u p}$ at frequency $f_{u p}>f_{\text {down }}$ by means of frequency scaling prediction methods is then the key point in the design of ULPC techniques.

Two different rain attenuation frequency scaling prediction methods are currently recommended in ITU-R P.618-12 [7]:

- Section 2.2.1.3.1 of ITU-R P.618-12 which provides a method of predicting the statistical variation of the rain attenuation at frequency $f_{u p}$ conditioned on the rain attenuation at frequency $f_{\text {down }}$. It is often referred to instantaneous frequency scaling (IFS) as it predicts the instantaneous uplink rain attenuation distribution based on the measured instantaneous downlink rain attenuation.

- Section 2.2.1.3.2 of ITU-R P.618-12 which provides a simplified method of predicting the equiprobable rain attenuation at frequency $f_{u p}$ conditioned to the rain attenuation at frequency $f_{\text {down }}$. It is often referred to long-term frequency scaling as it predicts the uplink rain attenuation based on knowledge of the downlink rain attenuation at the same probability of exceedance.

\subsection{Instantaneous frequency scaling}

The model has been first proposed by [20] and upgraded in [21]. It relies on two strong hypotheses:

- The probability distributions of rain attenuation on the up-link $A_{u p}$ and on the downlink $A_{\text {down }}$ conditioned to the presence of rain attenuation on the links are log-normal distributions with parameters sets $\left(\mu_{u p}, \sigma_{u p}\right)$ and $\left(\mu_{\text {down }}, \sigma_{\text {down }}\right)$ respectively for the up-link and the down-link, meaning:

$$
\left\{\begin{array}{l}
P\left(A_{u p}>a_{u p} \mid A_{u p}>0\right)=Q\left(\frac{\ln \left(a_{u p}\right)-\mu_{u p}}{\sigma_{u p}}\right) \\
P\left(A_{\text {down }}>a_{\text {down }} \mid A_{\text {down }}>0\right)=Q\left(\frac{\ln \left(a_{\text {down }}\right)-\mu_{\text {down }}}{\sigma_{\text {down }}}\right)
\end{array}\right.
$$

where $Q(x)=\frac{1}{\sqrt{2 \pi}} \int_{x}^{\infty} \mathrm{e}^{-\frac{t^{2}}{2}} \mathrm{~d} t$

- The probability distribution of rain attenuation at frequency $f_{u p}$ conditioned to the rain attenuation at frequency $f_{\text {down }}$ is a log-normal distribution with parameters set $\left(\mu_{\text {up/down }}, \sigma_{\text {up/down }}\right)$, meaning: 


$$
P\left(A_{u p}>a_{u p} \mid A_{\text {down }}=a_{\text {down }}\right)=Q\left(\frac{\ln \left(a_{u p}\right)-\mu_{u p / d o w n}}{\sigma_{u p / d o w n}}\right)
$$

Consequently, there is a correlation coefficient $\rho$ between the two underlying Gaussian processes $G_{u p}$ and $G_{d o w n}$ of the rain attenuation processes $A_{u p}$ and $A_{d o w n}$, respectively. Mathematically, it leads to:

$$
\left\{\begin{array}{l}
G_{u p}=\frac{\ln \left(A_{u p}\right)-\mu_{u p}}{\sigma_{u p}} \\
G_{d o w n}=\frac{\ln \left(A_{\text {down }}\right)-\mu_{\text {down }}}{\sigma_{\text {down }}} \\
\rho=E\left[G_{u p} G_{\text {down }}\right]
\end{array}\right.
$$

where $\mathrm{E}[]$ is the expectation operator. So, it is straightforward that:

$$
G_{u p}=\rho G_{d o w n}+\sqrt{1-\rho^{2}} n
$$

where $n$ is a White Gaussian noise with zero mean and unit variance. Using (10), (11) leads to:

$$
\ln \left(A_{u p}\right)=\rho \frac{\sigma_{u p}}{\sigma_{d o w n}} \ln \left(A_{d o w n}\right)+\left(\mu_{u p}-\rho \mu_{d o w n} \frac{\sigma_{u p}}{\sigma_{d o w n}}\right)+\sigma_{u p} \sqrt{1-\rho^{2}} n
$$

Parameters set $\left(\mu_{\text {up/down }}, \sigma_{\text {up/down }}\right)$ in (9) can then be retrieved from (12):

$$
\begin{aligned}
& \mu_{\text {up / down }}=\rho \frac{\sigma_{u p}}{\sigma_{\text {down }}} \ln \left(a_{\text {down }}\right)+\left(\mu_{u p}-\rho \mu_{\text {donw }} \frac{\sigma_{u p}}{\sigma_{\text {down }}}\right) \\
& \sigma_{\text {up/down }}=\sigma_{u p} \sqrt{1-\rho^{2}}
\end{aligned}
$$

Finally, based on data collected in Spino d'Adda during the ITALSAT experiment and in Louvain la Neuve, Lessive and Darmstadt during the OLYMPUS experiment, [22] has proposed a generic modelling of $\rho$ :

$$
\xi=\sqrt{1-\rho^{2}}=0.19\left(\frac{f_{u p}}{f_{\text {down }}}-1\right)^{0.57}
$$

which is the current parameterization of Section 2.2.1.3.1 of ITU-R P.618-12 recommended for frequency scaling up to $55 \mathrm{GHz}$.

\subsection{Long-term frequency scaling}


The long-term rain attenuation frequency scaling prediction method described in Section 2.2.1.3.2 of ITU-R P.618-12 is an empirical model. It gives an attenuation ratio directly as a function of frequency (in the range 7 to $55 \mathrm{GHz}$ ) and attenuation:

$$
A_{u p}=A_{\text {down }}\left(\varphi_{u p} / \varphi_{\text {down }}\right)^{1-H\left(\varphi_{\text {down }} \varphi_{u p}, A_{\text {down }}\right)}
$$

where:

$$
\begin{aligned}
& \varphi(f)=\frac{f^{2}}{1+10^{-4} f^{2}} \\
& H\left(\varphi_{\text {down }}, \varphi_{\text {up }}, A_{\text {down }}\right)=1.12 \times 10^{-3}\left(\varphi_{\text {up }} / \varphi_{\text {down }}\right)^{0.5}\left(\varphi_{\text {down }} A_{\text {down }}\right)^{0.55}
\end{aligned}
$$

$A_{\text {down }}$ and $A_{u p}$ are the equiprobable values of the rain attenuation at frequencies $f_{\text {down }}$ and $f_{u p}$ $(\mathrm{GHz})$, respectively.

\subsection{Tests of the prediction methods}

The data presented in Section III are used to test the two above models. As a reminder, ASTRA 3B and Alphasat satellites are respectively located at $23.5^{\circ}$ and $25^{\circ}$ of longitude giving almost the same slant path $\left(0.6^{\circ}\right.$ difference in elevation, and 1.90 difference in azimuth). The frequency scaling analysis it then appropriate.

\subsubsection{Parameterization}

According to (13), it is interesting to have a look on the experimental mean and standard deviation of the log-attenuation at $39.4 \mathrm{GHz}$ conditioned to the attenuation at 20.2 GHz. The model can be considered as valid if:

- there is a linear relationship between the mean of the log-attenuation at $39.4 \mathrm{GHz}$ and the log-attenuation at $20.2 \mathrm{GHz}$,

- the standard deviation of the log-attenuation at $39.4 \mathrm{GHz}$ is independent of the attenuation at $20.2 \mathrm{GHz}$.

This is observed on the results of Figure 8, thus validating the theoretical framework of the prediction method described in Section 4.1. The attenuation values at $20.2 \mathrm{GHz}$ have been voluntarily truncated in the range 1 to $7 \mathrm{~dB}$ because, first, calibration issues can impact the results at very low attenuation levels and, second, the attenuation at $39.4 \mathrm{GHz}$ could be higher than the dynamic range of the Q-band beacon receiver $(\sim 30 \mathrm{~dB})$ when attenuation at $20.2 \mathrm{GHz}$ is higher than $7 \mathrm{~dB}$.

The optimal experimental value of the correlation coefficient $\rho$ is estimated using the standard deviation of the log-attenuation at $39.4 \mathrm{GHz}$ (see (13b)). This extracted value of $\rho$ is then used to compute the mean of the log-attenuation at $39.4 \mathrm{GHz}$ conditioned to the attenuation at $20.2 \mathrm{GHz}$ (see (13a)). It experimentally leads to $\rho=0.9881$ (for information 
ITU-R P.618-12 gives $\rho=0.9828$ ) which is equivalent to $\xi=0.1536$ (ITU-R P.618-12 gives $\xi=0.1846$ ). Finally, all the results are reported in Figure 8.

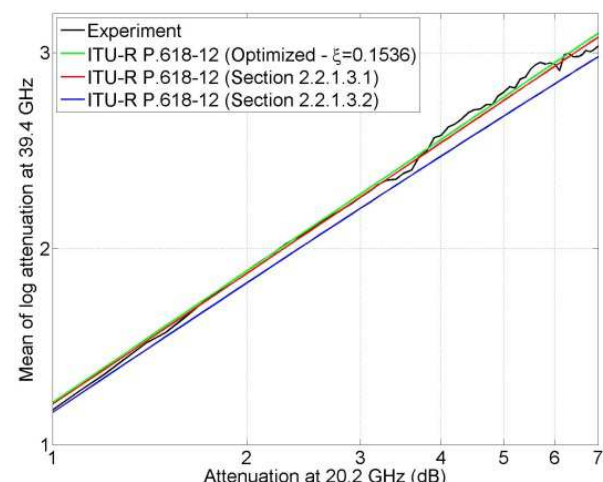

(a)

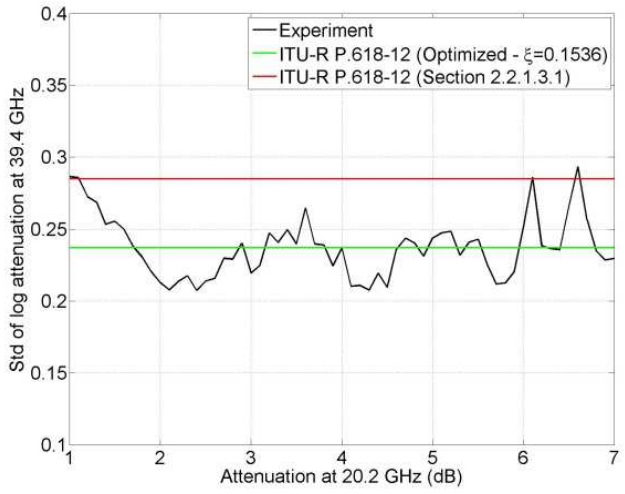

(b)

Figure 8. Mean (a) and standard deviation (b) of the log-attenuation at $39.4 \mathrm{GHz}$ conditioned to the attenuation at $20.2 \mathrm{GHz}$

\subsubsection{Comparisons}

The final comparisons with both long-term and instantaneous frequency scaling prediction methods are reported in Figure 9 and Figure 10, respectively. On a long-term basis, it could be observed a slight underestimation of the method described in Section 2.2.1.3.2 of ITU-R P.618-12, while a perfect agreement is reached using the method described in Section 2.2.1.3.1 of ITU-R P.618-12. On an instantaneous basis, as expected, the optimal parameterization of the method described in Section 2.2.1.3.1 of ITU-R P.61812 performs better than the generic parameterization (i.e. using (14)) which, however, still exhibits an appropriate behaviour even for very low probability levels.

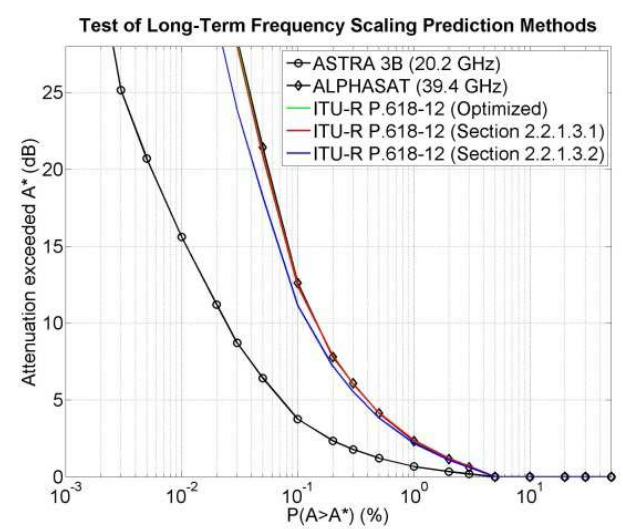

Figure 9. Test of long-term frequency scaling prediction methods 


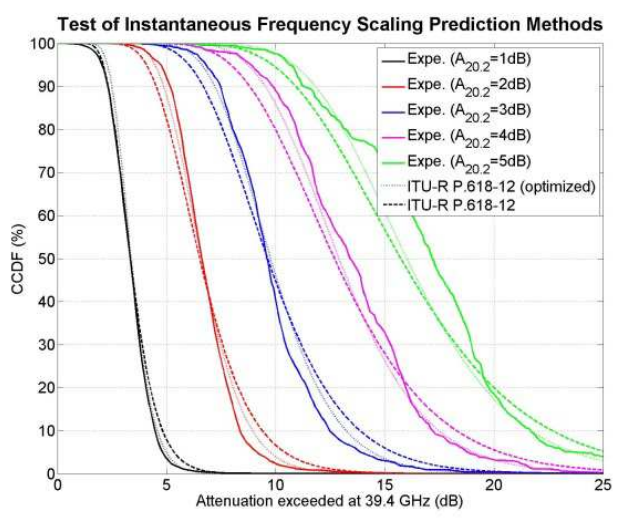

(a)

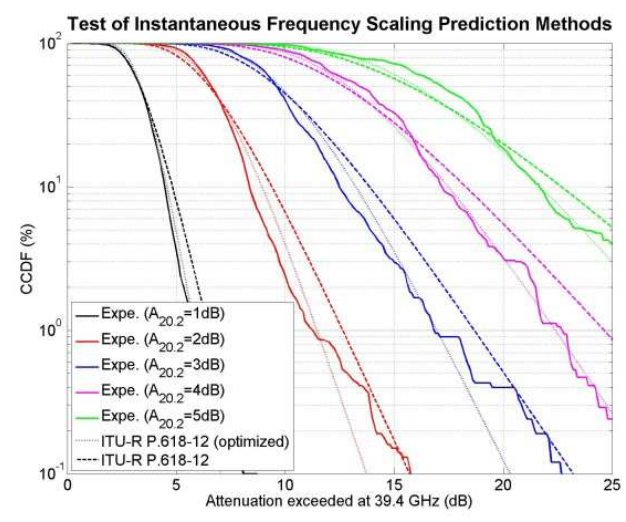

(b)

Figure 10. Test of instantaneous frequency scaling prediction methods

\section{CONCLUSION}

Since June 2015, ONERA has been conducting concurrent $\mathrm{Ka}$ and $\mathrm{Q}$ band propagation experiments in Toulouse, France. Two indoor beacon receivers, designed and built at ONERA, are used: the first one is able to record the $20.2 \mathrm{GHz}$ beacon signal of ASTRA 3B, the second one is pointed towards the $39.4 \mathrm{GHz}$ beacon of Alphasat. Two full years of measurements (from June 2015 to May 2017) have been recorded and processed so far. Daily time series of rain and total attenuation have been extracted. As the availabilities of valid data are around $95 \%$, reliable statistical analyses have been performed. A high month-to-month variability can be noticed with more than $20 \mathrm{~dB}$ difference of rain attenuation at $39.4 \mathrm{GHz}$ from the most favourable month to the worst. Using concurrent measurements at 20.2 and $39.4 \mathrm{GHz}$, the two prediction methods of frequency scaling recommended in ITU-R P.618-12 have been tested. On a long-term basis, a slight underestimation of the method described in Section 2.2.1.3.2 of ITU-R P.618-12 can be noticed, while a perfect agreement is reached using the method described in Section 2.2.1.3.1 of ITU-R P.618-12. On an instantaneous basis, the method described in Section 2.2.1.3.1 of ITU-R P.618-12 shows very good performances. The ASTRA 3B and Alphasat propagation experiments are still on-going and the testing activity of frequency scaling prediction methods needs to be consolidated from the expected new results.

\section{ACKNOWLEDGMENT}

This work has been carried out under ONERA internal funding and under CNES R\&T studies: R-S13/TC-0005-048- "Dual-Polarization Propagation Experiment at Ka And Q Bands" and R-S16/TC-0005-084 - "Improvement of Propagation Models at Ka and Q/V Bands over Europe". 


\section{REFERENCES}

1. O. Koudelka, "Q/V-band communications and propagation experiments using ALPHASAT", Acta Astronautica, vol. 69, no. 11-12, pp. 1029-37, December 2011.

2. T. Rossi et al., "Satellite Communication and Propagation Experiments Through the Alphasat Q/V Band Aldo Paraboni Technology Demonstration Payload”, IEEE A\&E Systems Magazine, vol. 31, issue 3, pp. 18-27, March 2016, DOI. No. 10.1109/MAES.2016.150220.

3. C. Riva et al., "The Aldo Paraboni Scientific Experiment: the preparation and plans for a European measurement campaign", Proc. of 20th Ka and Broadband Communications Navigation and Earth Observation Conference, October 1-3 2014, Salerno, Italy.

4. X. Boulanger et al., "Four Years of Total Attenuation Statistics of Earth-Space Propagation Experiments at Ka-Band in Toulouse," IEEE Trans. Ant. Prop., vol. 63, no. 5, May 2015.

5. X. Boulanger et al., "Small and large scale site diversity experiment at Ka-band in the south of France", Int. J. Satell. Commun. Network. (2016) Published online in Wiley Online Library (wileyonlinelibrary.com). DOI: 10.1002/sat.1190.

6. X. Boulanger, and L. Castanet, "Alphasat Propagation Experiment in Toulouse: Two years of measurements", Proc. of 23rd Ka and Broadband Communications Conference, October 16-19 2017, Trieste, Italy.

7. ITU-R Recommendations P.618-12, "Propagation data and prediction methods required for the design of Earth-space telecommunication systems", Geneva, July 2015.

8. http://www.meteofrance.com/climat/france/toulouse/31069001/normales

9. J. Lemorton et al., "A new opportunity for EHF propagation experiments: the EXPRESS campaign with the satellite STENTOR", Int. J. Satell. Commun., vol. 19, pp. 347-362, 776 May/Jun. 2001.

10. F. Barbaliscia (general editor), "Volume 3: reference book on radiometry and meteorological measurements", OPEX, Second Workshop of the OLYMPUS Propagation Experimenters, ESA WPP-083, Noordwijk, 8-10 November 1994.

11. C. Riva et al., "COST IC0802 Handbook on measurements and products", 2014.

12. F. Solheim et al., "Radiometric Profiling of Temperature, Water Vapor, and Cloud Liquid Water using Various Inversion Methods", Rad. Sci., 33, pp. 393-404, 1998.

13. F. Solheim et al., "Microwave Radiometer for Passively and Remotely Measuring Atmospheric Temperature, Water Vapor and Cloud Liquid Water Profiles", US Army White Sands Missile Range Final Report, 52 pp., 1996.

14. F. T. Ulaby et al., "Microwave Remote Sensing Active and Passive", Vol.1, Addison-Wesley, 288302, 1981.

15. D. J. Fixsen, "The Temperature of the Cosmic Microwave Background", The Astrophysical Journal 707(2):916-920. doi:10.1088/0004-637X/707/2/916, December 2009.

16. ITU-R SG3, Working Party 3M, "On the processing of tipping bucket rain gauge data for study group 3 experimental database", Revision 1 to Fascicle 3M/FAS/8, October 2017.

17. J. P. V. Poiares-Baptista, and S. E. Dinwiddy, "Up-link power control on a high-availability satellite link operating at $30 \mathrm{GHz}$ ", IEE Colloquium on Fade Countermeasures and Channel Restoration on Earth-Space Links, 2 March 1990; 4/1-4/8.

18. K. T. Lin et al., "Results of an experiment to demonstrate the effectiveness of open-loop up-link power control for Ku-band satellite links", $8^{\text {th }}$ International Conference on Antennas and Propagation, Edinburgh vol. 1, 1993; 202-205.

19. A. W. Dissanayake, "Application of open-loop uplink power control in Ka-band satellite links", Proceedings of the IEEE 1997; 85(6):959-969.

20. S. Bertorelli S., A. and Paraboni, "Modelling of short-term frequency scaling for rain attenuation using ITALSAT data", Int. J. Satell. Commun. Network. 2007; 25:251-262. DOI: 10.1002/sat.867.

21. Castanet L., Bourgoin J., Pradie M., Bertorelli S., Paraboni A., Martellucci A. : "Characterisation and modelling of instantaneous frequency scaling in $20-50 \mathrm{GHz}$ band", $3^{\text {rd }}$ CNES workshop on Earthspace propagation, Toulouse, France, September 2006. 
22. ITU-R SG3, Working Party 3M, "Information document about frequency scaling in Recommendation ITU-R P.618-10. Instantaneous frequency scaling of rain attenuation", Input Document 3M/35 from France, Italy, and European Space Agency, Geneva.

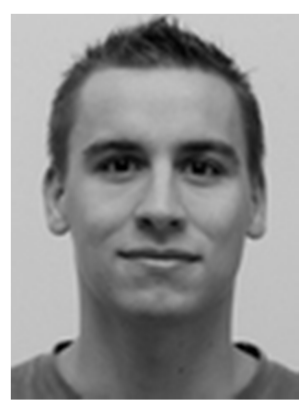

Xavier Boulanger was born in Montpellier, France, in 1986. He received his Diploma of Engineering in Electronics and Digital Communications from the Ecole Nationale de l'Aviation Civile (ENAC), Toulouse, France, in 2010. He received his PhD degree in propagation and signal processing in 2013 from Institut Supérieur de l'Aéronautique et de l'Espace (ISAE), Toulouse, France. In 2013, he joined the French Aerospace Lab (ONERA) as Research Engineer, where he is currently working on propagation channel modelling for Earth-Space communication systems. He was involved in various working groups dealing with Earth-space propagation modelling such as COST IC0802, and ITU-R Study Group 3.

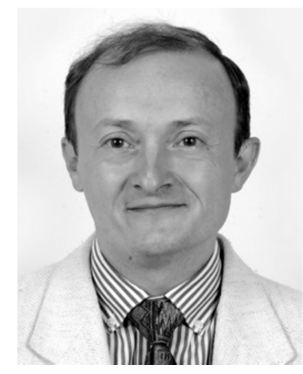

Laurent Castanet works as Research Engineer in the radiocommunication field at ONERA, Toulouse, France. His main research interests are Earth-Space propagation and Fade Mitigation Techniques. He is the Head of the Radio-Communication and Propagation research unit of the Electromagnetics and Radar department of ONERA.

He has been involved in propagation and radiocommunication studies for satellite systems. He is involved more particularly in studies related to the dynamics of the propagation channel, to the development and validation of propagation models in temperate and tropical areas as well as to Fade Mitigation Techniques for $\mathrm{Ka}$ and $\mathrm{Q} / \mathrm{V}$ band systems for CNES, ESA, European Industry and French MoD.

He was a French expert in COST 255, COST 280 and COST IC-0802 European projects, in which he worked on the development of advanced models of the space-time variability of the propagation channel and on the design, simulation and optimisation of adaptive Fade Mitigation Techniques. He represented ONERA in the European Network of Excellence "SatNEx", in which he chaired the "Channel modelling and propagation impairment simulation" Joint Activity. He is also the Head of the French Delegation to ITU-R Study Group 3 which deals with radiowave propagation.

He teaches radiowave propagation in French Engineering Schools and University (ISAE, Telecom Bretagne, University Paul Sabatier) as well as link analysis at EUROSAE Training institution, Toulouse, France. 


\begin{tabular}{|c|c|c|c|}
\cline { 2 - 4 } \multicolumn{1}{c|}{} & $\begin{array}{c}\mathbf{1}^{\text {st }} \text { Year } \\
\text { (June 2015- } \\
\text { May 2016) }\end{array}$ & $\begin{array}{c}\mathbf{2}^{\text {nd }} \text { Year } \\
\text { (June 2016- } \\
\text { May 2017) }\end{array}$ & $\begin{array}{c}\text { Total } \\
\text { (June 2015- } \\
\text { May 2017) }\end{array}$ \\
\hline Rain attenuation at 39.4 GHz & 96.19 & 97.47 & 96.83 \\
\hline Total attenuation at 39.4 GHz & 94.65 & 97.47 & 96.06 \\
\hline Concurrent rain attenuation at 39.4 GHz and rainfall rate & 93.73 & 96.38 & 95.05 \\
\hline Concurrent rain attenuation at 20.2 and 39.4 GHz & 96.14 & 95.11 & 95.62 \\
\hline Concurrent total attenuation $\mathbf{2 0 . 2}$ and 39.4 GHz & 94.65 & 95.11 & 94.88 \\
\hline
\end{tabular}

Table 1. Availabilities of valid data. 